\title{
Nonlinear Control Structure of Grid Connected Modular Multilevel Converters
}

\author{
A. Hajizadeh ${ }^{1}$, L.E.Norum ${ }^{2}$, A.Ahadpour Shal ${ }^{3}$ \\ ${ }^{1}$ Department of Energy Technology, Aalborg University \\ Campus of Esbjerg - Niels Bohr Vej 8, 6700 (Denmark) \\ E-mail: aha@et.aau.dk \\ ${ }^{2}$ Department of Electric Power Engineering, Norwegian University of Science and Technology \\ O. S. Bragstads plass 2, Trondheim, Norway \\ E-mail: norum@ntnu.no \\ ${ }^{3}$ Faculty of Electrical Engineering and Information Technology, RWTH-Aachen University, Germany \\ E-mail: alireza.ahadpour@rwth-aachen.de
}

\begin{abstract}
This paper implements nonlinear control structure based on Adaptive Fuzzy Sliding Mode (AFSM) Current Control and Unscented Kalman Filter (UKF) to estimate the capacitor voltages from the measurement of arm currents of Modular Multilevel Converter (MMC). UKF use nonlinear unscented transforms in the prediction step in order to preserve the stochastic characteristics of a nonlinear system. In order to design adaptive robust control strategy and nonlinear observer, mathematical model of MMC using rotating $\mathrm{d}-\mathrm{q}$ theory has been used. Digital time-domain simulation studies are carried out in the Matlab/Simulink environment to verify the performance of the overall proposed control structure during different case studies.
\end{abstract}

\section{Key words}

Nonlinear Control, Kalman Filter, Nonlinear observer, Modular Multilevel Converter

\section{Introduction}

Power electronic converters should have a high reliability, high efficiency, good harmonic performance, low cost, and a small footprint. Modular Multilevel Converters (MMC) are promising solutions for high power converters since they allow the combination of excellent harmonic performance and low switching frequencies [1-2]. Modular Multilevel Converter (MMC) is a new form of multilevel converter and it is a big improvement in this area especially for interconnection of High Voltage DC (HVDC) transmission system and large wind farms [3]. Regarding to the many control complexities in MMC due to modularity, high number of sub modules parameters and limitation of measurements of all voltages and currents, some control strategies have been present by literatures which are categorized as control strategies of MMC for submodule (SM) voltage balancing [4-5], circulating current suppressing [6-7] and AC side current control for grid connected application [8]. In most presented works, the analysis of MMC during unbalanced grid conditions has not been investigated. Moreover, a comprehensive dynamic model for MMC that combines symmetrical positive and negative sequences is needed to design advanced control structures. Therefore, in continuing of the proposed control strategies and for making the MMC performance robust during unbalanced grid conditions with unknown constant parameters, an non-linear control structure for grid connected MMC is proposed. The control structure is composed adaptive fuzzy sliding mode (AFSM) control and non-linera observer based on unscented kalman filter (UKF). Due to high nonlinearities in model of MMC during unbalanced grid condition, using of fuzzy sliding mode control is proposed. An adaptive learning method also is added to this structure to train the parameters of controller realtime. In this structure, the capacitance value of the cell capacitors as a uncertain parameter which should be measured carefully. In order to design the observer, from the developed nonlinear model of MMC, a nonlinear observer based on unscented kalman filter (UKF) is employed to estimate the capacitor voltage of each cell. The unscented transform is a nonlinear transformation that propagates the mean and covariance through a nonlinear function. This method is based on a set of chosen sample point, known as sigma points, and preserves the nonlinear nature of the system. One way to handle nonlinear models and transformations is to combine kalman filter with the unscented transform to obtain the unscented kalman filter [10]. This approach is very promising in power electronic converters because their nonlinear model is known with sufficient accuracy. The first measurement considered is the arm currents which are the controlled variables and makes it possible to estimate the capacitor voltages. Afterward, it is necessary to know the dc-link voltage state, because of the capacitors voltages references depend on it. Finally, some illustrative simulation results of MMC are demonstrated to confirm the feasibility of the proposed PWM algorithm as well as the designed observer. This 
paper is organized as follow. Section II presents the MMC model. The discrete UKF and observer are described in section III. In section IV, the control design is proposed. Section V shows the simulation results. Conclusion is given in section VI.

\section{Modelling of Modular Multilevel Converter}

The typical structure of a MMC is shown in Fig. 1, and the configuration of a SubModule (SM) is given in Fig. 2. Each $\mathrm{SM}$ is a simple chopper cell composed of two IGBT switches $\left(T_{1}\right.$ and $\left.T_{2}\right)$, two anti-parallel diodes $\left(D_{1}\right.$ and $D_{2}$ ) and a capacitor $C$ [2-3].

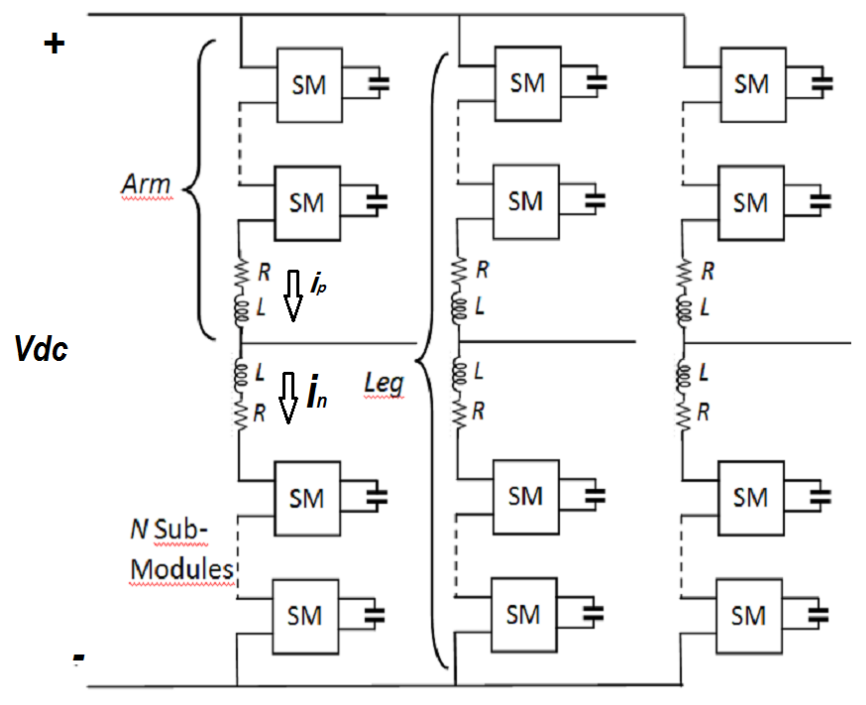

Fig.1 The typical structure of a MMC

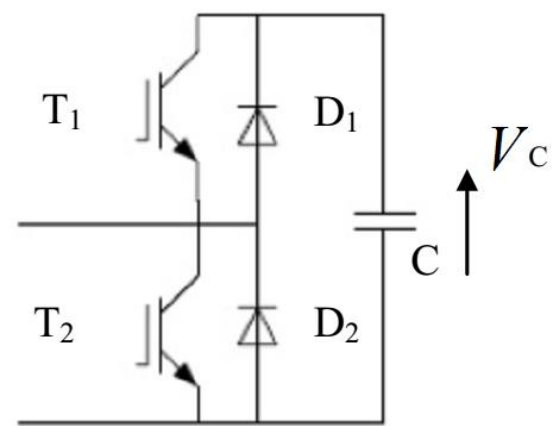

Fig.2 Configuration of a SubModule (SM) fo MMC

From Figs.1 and 2, the mathematical equations describing the dynamic behavior of a N-cells MMC are expressed as follows:

$$
\begin{aligned}
& \frac{d i_{p}}{d t}=\frac{1}{L}\left[\frac{V_{d c}}{2}-\sum_{i=1}^{N}\left(u_{i} \cdot V_{C_{i-n}}\right)-R i_{p}-V_{a}\right] \\
& \frac{d i_{n}}{d t}=\frac{1}{L}\left[\frac{V_{d c}}{2}-\sum_{i=N+1}^{2 N}\left(u_{i} \cdot V_{C_{i}-l}\right)-R i_{n}+V_{a}\right] \\
& \frac{d V_{C_{i-u}}}{d t}=\frac{1}{C}\left(i_{p} \cdot u_{i}\right) \quad i=1, \ldots ., N \\
& \frac{d V_{C_{i-l}}}{d t}=\frac{1}{C}\left(i_{n} \cdot u_{i}\right) \quad i=N+1, \ldots ., 2 N
\end{aligned}
$$

where $i_{p}, i_{n} u_{i}, V_{C i_{\_} u} V_{C i_{-} l}$, and $V_{a}$ are upper/lower are currents, gating signal of upper gate, upper and lower capacitor voltages of the $i$-th cell, and phase $a$ voltage respectively.

\section{Unscented Kalman Filter (UKF) and Observer Design}

The KF was originally developed for linear systems but later applied to nonlinear systems using the linearized or extended kalman filter (EKF) and its performance is acceptable if the system nonlinearity is not severe. Its simplicity, together with the popularity of the KF, makes it the most widely applied nonlinear state estimator [10]. The discrete KF uses the first two statistical moments and updates them with time. This is the key idea when combining the UT and KF to obtain UKF. The UKF is basically the discrete KF in which a UT is used to obtain the mean and covariance updates. The UKF as presented here is a simplified UKF which is suitable for estimation of IM states. In general, the observation model can also be nonlinear, and all parameters and functions can be time varying. Moreover, the UKF can be extended to the case of nonadditive noise [10]. The discrete-time nonlinear system is given as follow:

$x(k+1)=f(x(k), u(\mathrm{k}))+\omega(\mathrm{k})$

and a linear measurement model

$$
y(k)=H \cdot x(k)+v(k)
$$

where $x(k)$ is an $\mathrm{n} \times 1$ state vector, $y(k)$ is an $\mathrm{m} \times 1$ measurement vector, $H$ is the measurement matrix $(\mathrm{m} \times$ $\mathrm{n}$ ), and $f(x(k), u(k))$ is a known nonlinear state transition vector. We assume that the process noise $w(k)$ is white and zero mean with covariance matrix $Q$ and the measurement noise $v(k)$ is also white and zero mean with covariance matrix $R$. We also have estimates of the initial state $x^{+}(0)$ and the initial error covariance matrix $P^{+}(0)$. The iterations in the classic KF consist of a prediction step followed by a correction step. For the correction step, we use the discrete KF equations

$$
\begin{aligned}
& K(k+1)=P^{-}(k) \cdot H^{T} \cdot\left(H \cdot P^{-}(k) \cdot H^{T}+R\right)^{-1} \\
& x^{+}(k)=x^{-}(k)+K(k) \cdot\left(y(k)-H \cdot x^{-}(k)\right) \\
& P^{+}(k)=(I-K(k) \cdot H) \cdot P^{-}(k)
\end{aligned}
$$

where $K(k)$ is the Kalman gain.

The prediction step in the $\mathrm{KF}$ is the projection of the mean $\mathrm{x}^{+}(\mathrm{k})$ and covariance $\mathrm{P}^{+}(\mathrm{k})$ in time using the state equation (recall that the KF estimates are unbiased). For the nonlinear system of (1), the state equation is a nonlinear transformation of a stochastic input $x(k)$. Hence, we can use the UT to obtain the mean $\mathrm{x}^{-}(\mathrm{k}+1)$ and covariance $\mathrm{P}^{-}(\mathrm{k}+1)$ of its output. To use the discrete $\mathrm{KF}$, the MMC model is discretized by solving the system's state equation to determine the states at the sampling instants. The resulting system is: 


$$
\begin{aligned}
& i_{p}(\mathrm{k}+1)=i_{p}(\mathrm{k})+\frac{T_{s}}{L}\left[\frac{V_{d c}}{2}-\sum_{i=1}^{N}\left(u_{i}(\mathrm{k}) \cdot V_{C_{i-u}}(\mathrm{k})\right)-R i_{p}(\mathrm{k})-V_{a}(\mathrm{k})\right] \\
& i_{n}(\mathrm{k}+1)=i_{n}(\mathrm{k})+\frac{T_{s}}{L}\left[\frac{V_{d c}}{2}-\sum_{i=N+1}^{2 N}\left(u_{i}(\mathrm{k}) \cdot V_{C_{i-l} l}(\mathrm{k})\right)-R i_{n}(\mathrm{k})+V_{a}(\mathrm{k})\right] \\
& V_{C_{i-u}}(\mathrm{k}+1)=V_{C_{i-u}}(\mathrm{k})+\frac{T_{s}}{C}\left(i_{p}(\mathrm{k}) \cdot u_{i}(\mathrm{k})\right) \quad i=1, \ldots ., N \\
& V_{C_{i-l} l}(\mathrm{k}+1)=V_{C_{i-l} l}(\mathrm{k})+\frac{T_{s}}{C}\left(i_{n}(\mathrm{k}) \cdot u_{i}(\mathrm{k})\right) \quad i=N+1, \ldots, 2 N
\end{aligned}
$$

Now, the state space model of MMC is related to the UKF structure. The state vector is chosen as

$$
X=\left[\begin{array}{llll}
i_{p} & i_{n} & V_{C_{i_{-} u}} & V_{C_{i_{-} l}}
\end{array}\right]
$$

The arm currents are the measured output, and the measurement is

$$
H=\left[\begin{array}{llll}
1 & 0 & 0 & 0 \\
0 & 1 & 0 & 0
\end{array}\right]
$$

Thus, the measurement model is linear as in (3). Next, the UKF observer is implemented using (3)-(4) to accurately estimate the capacitor voltages.

\section{Adaptive Fuzzy Sliding Mode Control Design}

For the control plant given in (5), the sliding mode control law can be derived in order to reach a desired value of the output currents at sampling instants $t=k T$, the following condition has to fulfilled.

$$
\left[\begin{array}{c}
S_{p}(\mathrm{k}) \\
S_{n}(\mathrm{k})
\end{array}\right]=\left[\begin{array}{c}
i_{p}(\mathrm{k}) \\
i_{n}(\mathrm{k})
\end{array}\right]-\left[\begin{array}{l}
i_{p}^{r e f} \\
i_{n}^{r e f}
\end{array}\right]
$$

Where $i_{p}^{r e f}$ and $i_{n}^{r e f}$ are the specified current vector commands and $S(k)=S(x(k))$ represents the discrete-time sliding-mode surface control and $x(k)$ represents the discrete state vector of the converter.

The sliding-mode control theory has been widely employed to control nonlinear systems, especially the systems that have model uncertainty and external disturbance [11]. Robustness is the best advantage of a sliding-mode control. It employs a noncontiguous control effort to drive the system toward a sliding surface, and then switches on that surface. Theoretically, it will gradually approach the control objective, the origin of a phase plane. The fuzzy logic controller needs only simple computation and programming capacity to represent human control behavior, and, in recent years, has had widespread use in engineering applications. Since there is a lack of theoretical modeling and analysis for the FLC stability and robustness problem, the commercial industrial application has progressed slowly. The fuzzy system was used to approximate an optimal controller, which was adjusted by an adaptive law based on Lyapunov stability theory. However, this kind of direct adaptive law is limited to the nonlinear system with constant control gain. After that, the fuzzy direct control scheme was proposed [12]. This control architecture employed a fuzzy system to approximate an optimal controller that was designed based on the assumption that all the dynamics in the system were known, and then a fuzzy sliding controller was added to the adaptive controller for compensating the uncertainties and smoothing the control signal.

Define, $e$ is the phase plane variable. Then, the sliding surface on the phase plane can be defined as:

$$
\begin{aligned}
& s=\left(\frac{d}{d t}+l\right) e=\frac{d}{d t}\left[\begin{array}{l}
e_{1} \\
e_{2}
\end{array}\right]+\lambda\left[\begin{array}{l}
e_{1} \\
e_{2}
\end{array}\right] \\
& s=\left(X_{r e f}-A X(t)-g(t) u\right)+\lambda\left(X_{r e f}-X(t)\right)
\end{aligned}
$$

Based on the Lyapunov theorem, the sliding surface reaching condition is $s . \dot{s}<0$. If a control input $u$ can be chosen to satisfy this reaching condition, the control system will converge to the origin of the phase plane. From (5) and (9), it can be concluded that the variable $g(t)$ is always a positive/negative value for charging/discharging conditions. It can also be found that increases as decreases and vice versa in (7). If $s<0$, then increasing $\mathrm{u}$ will result in decreasing. When the condition is $s<0$, will decrease with the decreasing of $u$. Based on this qualitative analysis, the control input can be designed in an attempt to satisfy the inequality. Here, a new control strategy with an FLC-based direct adaptive controller is proposed, the control law of which is generated directly from the output of an FLC. The control voltage change for each sampling step is derived from fuzzy inference and defuzzification calculation instead of the equivalent control law derived from the nominal model at the sliding surface. It can diminish the chattering phenomenon of the traditional sliding-mode control. The controller design does not need a mathematical model and without constant gain limitation. The block diagram is shown in Fig. 3. An online parameters tuning algorithm is developed based on the steep descent rule. The consequent parameters of the FLC can be initially set to zero, then, the novel parameters tuning algorithm is used to adjust the consequent parameters to monitor the system control performance. The sliding surface variable is employed as the one-dimensional fuzzy input variable. The control law is the output of the FLC which is a function of $(A, s$, $g(t), e, t)$. Here, seven triangular membership functions are used for the fuzzy input $(s)$ and output $(u)$ variables. Since the proposed control strategy has on-line tuning algorithm for fuzzy rules adjustment, the number of rules is not critical.

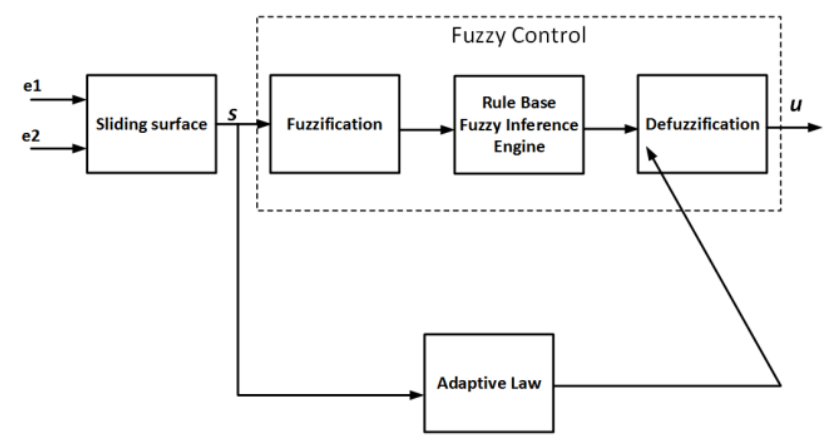

Fig.3 Control system block diagram of an Adaptive Fuzzy Sliding Mode Control

The minimum number of the rules is three for this control strategy. Here, seven fuzzy rules are employed in this 
control system to obtain appropriate dynamic response and control accuracy. The input membership functions are scaled into the range of -1 and 1 with equal span. The control law is derived from the fuzzy inference decision and defuzzification operation.

$$
u=\frac{\sum_{l}^{m} \mu^{j} \cdot U^{j}}{\sum_{l}^{m} \mu^{j}}=\frac{\sum_{l}^{m} \mu^{j} \cdot C^{j}}{\sum_{l}^{m} \mu^{j}}
$$

Where $m$ is the rules number and $C^{j}$ is the consequent parameter. Which can be initially set to zero as a zero fuzzy rule beginning. An adaptive rule is used to adjust or update it. The adaptive rule is derived from the steep descent rule to minimize the value of with respect to $C^{j}$. Then, the modification equation of the parameter is

$$
\dot{C}^{j}=-\eta \frac{\partial s(\mathrm{t}) \cdot \dot{s}(\mathrm{t})}{\partial C^{j}(\mathrm{t})}
$$

where $\eta$ is the adaptive rate parameter. Based on the chain rule, the above equation can be rewritten as

$$
\begin{aligned}
& \dot{C}^{j}=-\eta \frac{\partial s(\mathrm{t}) \cdot \dot{s}(\mathrm{t})}{\partial u(\mathrm{t})} \frac{\partial u(\mathrm{t})}{\partial C^{j}(\mathrm{t})} \\
& =-\eta g(\mathrm{t}) \cdot \mathrm{s}(\mathrm{t}) \frac{\partial u(\mathrm{t})}{\partial C^{j}(\mathrm{t})} \\
& =\theta s(\mathrm{t}) \frac{\mu^{j}(\mathrm{t})}{\sum_{l}^{m} \mu^{j}(\mathrm{t})}
\end{aligned}
$$

where the adaptive rate parameter $\eta$ and the system input parameter $g(t)$ are combined as a learning rate parameter $\theta$. Then, the central positions of the defuzzification membership functions can be regulated directly through the modification of consequent parameter, $C^{j}$. Hence, it achieves the objectives of online learning and fuzzy rules adjustment. This adaptive rule has two main contributions to this proposed model-free fuzzy slidingmode control. Firstly, it simplifies the implementation of fuzzy control without trial-and-error process for finding appropriate fuzzy rules. Secondly, this online adaptive rule also has the effect of improving the stability property and increasing the speed of reaching the sliding surface due to the steep descent rule.

\section{Simulation Results}

In order to evaluate the performance of the proposed control strategy and nonlinear observer performance, simulation results are carried out in MATLAB/Simulink software with the nominal parameters given in Table I. The set-points for the transferred real and reactive power at $\mathrm{t}=0$ are set to $300 \mathrm{~kW}$ and $0 \mathrm{kVar}$, respectively. A real power flow command is applied at $t=5 \mathrm{~s}$ by changing the reference of real power to $P_{r e f}=200 \mathrm{~kW}$.

In Fig.4, the estimated capacitor voltage has been illustrated. The three-phase line-line voltages are shown in Fig. 5. It clearly reveals the presence of eight levels of voltage, as expected. The charge and discharge of the capacitors cause the voltage levels to vary within acceptable limits. The eight capacitor voltages of phase A are presented in Fig.6. The sorting algorithm ensure that all the capacitors are fairly balanced at reference voltage $150 \mathrm{~V}$. They have a voltage variation of around $10 \%$ of this value.

TABLE I. Parmaters of MMC

\begin{tabular}{|c|c|}
\hline \multicolumn{2}{|c|}{ Nominal Parameters } \\
\hline DC link voltage & $600 \mathrm{~V}$ \\
\hline Rated line-line voltage & $600 \mathrm{~V}$ \\
\hline Number of Cells per arm & 4 \\
\hline Cell capacitance & $800 \mu \mathrm{F}$ \\
\hline Rated frequency & $50 \mathrm{~Hz}$ \\
\hline Carrier frequency & $1850 \mathrm{~Hz}$ \\
\hline Arm inductance & $5 \mathrm{mH}$ \\
\hline Arm resistance & $15 \mathrm{~m} \Omega$
\end{tabular}

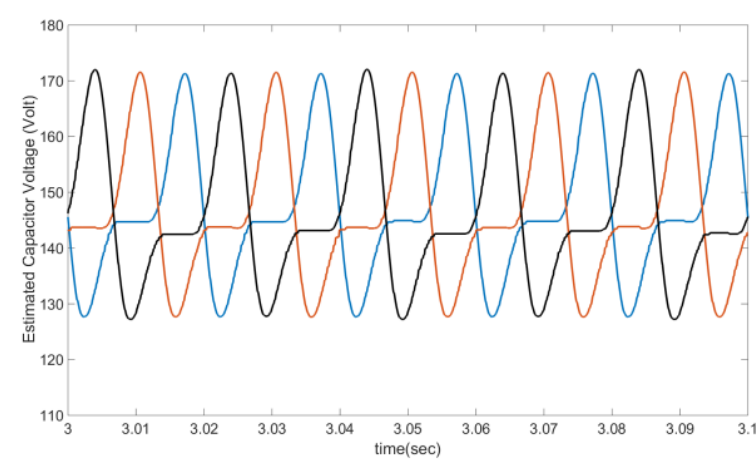

Fig.4. the estimated capacitor voltage

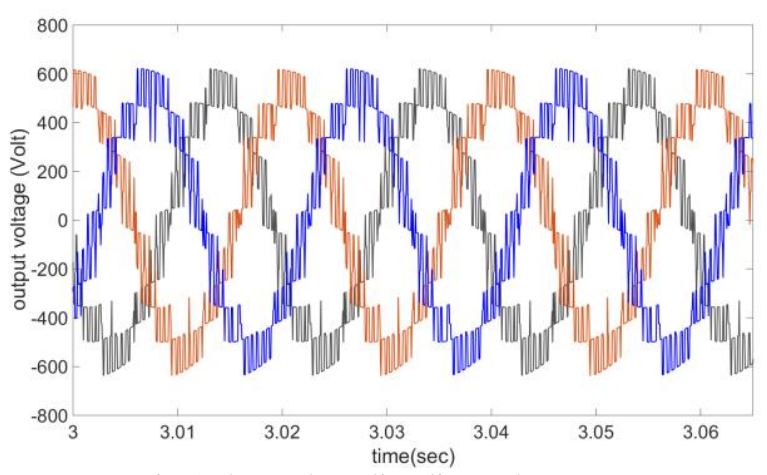

Fig.5. three-phase line-line voltages

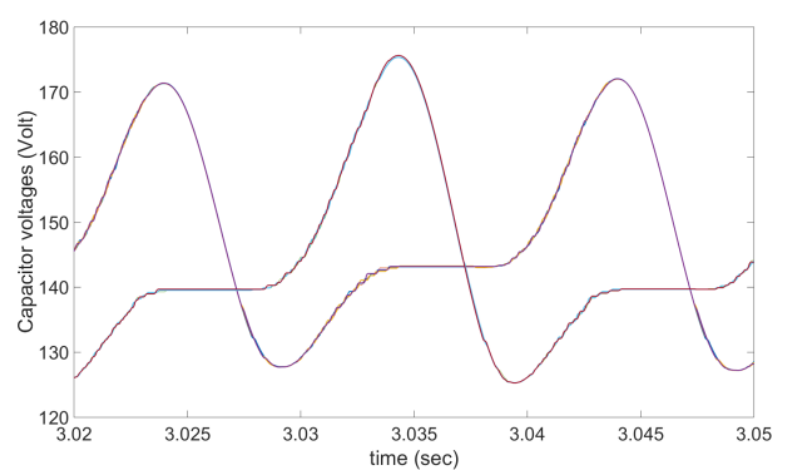

Fig.6. simulated eight capacitor voltages

In Fig.7, the voltage across the top cell of phase A is presented. It shows that the cell switches 18 times in a cycle. This leads to a switching frequency of 
approximately $1 \mathrm{kHz}$ for the IGBT, which is an acceptable at this rating. The arm currents and load current for phase A are illustrated in Fig.8 There is a circulating dc that flows through the arms and the dc bus. This dc current helps to maintain the power balance in the capacitors.

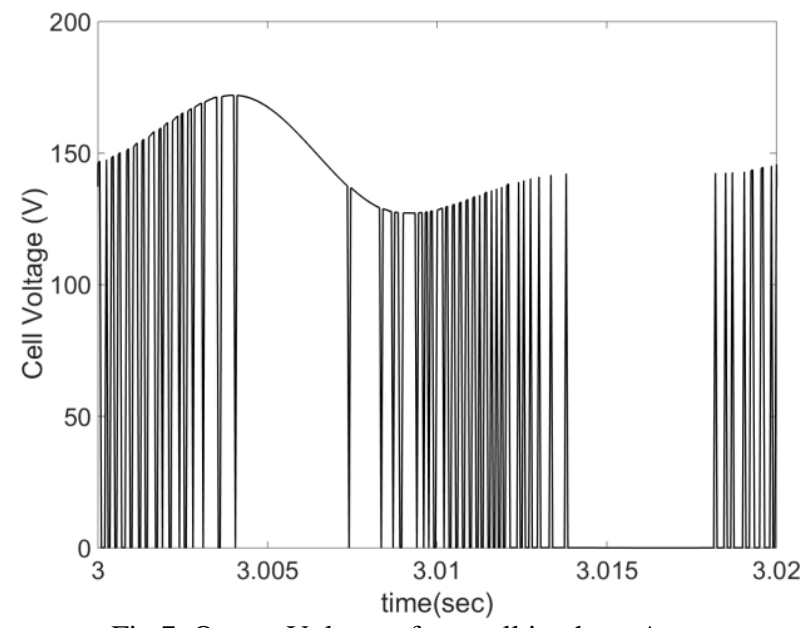

Fig.7. Output Voltage of top cell in phase A

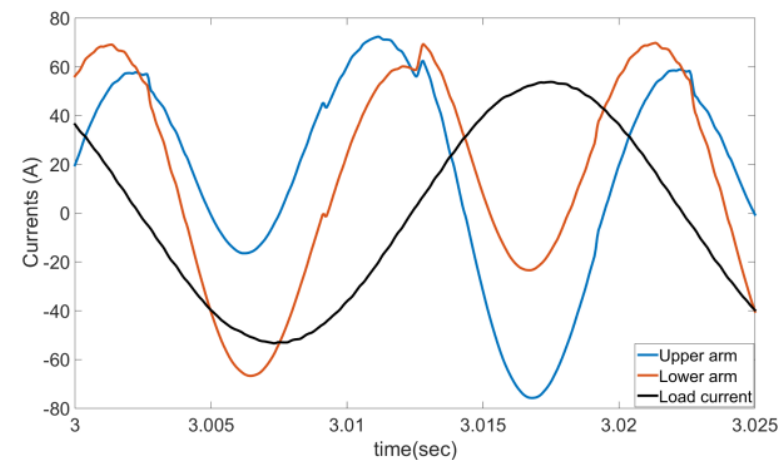

Fig.8. Upper/lower arm and load currents

Fig.9 shows the dynamic response of the MMC to the power flow command. It shows the corresponding changes in the transferred real and reactive power and there is an improvement in the steady-state and transient behavior of active power due to the implementation of proposed robust controller.
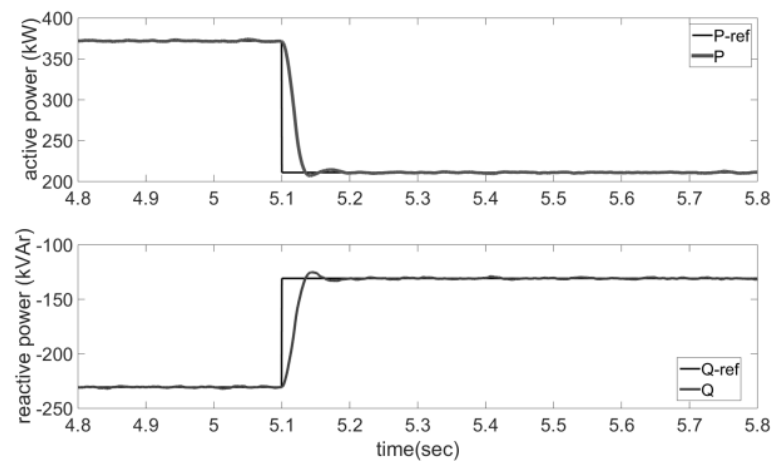

Fig.9. dynamic response of the MMC to the step power command.

The $d$ and $q$ axis current components are presented in Fig.10. As shown, the proposed current control strategy is capable to track the current references properly and it can overcome the drawback of the traditional decoupling control easily. Moreover, it is seen that there are no transient conditions in response of proposed controller.
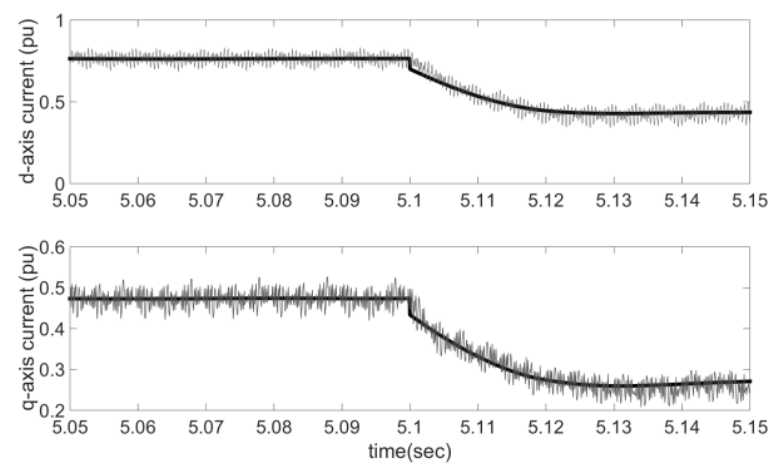

Fig.10. $d$ and $q$ axis current components

In order to evalautae the dynamic response of the proposed controller structure, another simulation case has been performed. In this case, the DC voltage control and reactive power reference control are presented. The ability of reactive power control was tested and the circullating current and voltage balancing control behavior during reactive power change were evaluated. At $\mathrm{t}=0.2 \mathrm{~s}$ the circullating current strategy control (CCSC) is enabled and at $\mathrm{t}=0.4 \mathrm{sec}$ the reactive power has a step change from 0 p.u. to 0.33 p.u. The results are shown in Fig. 11.
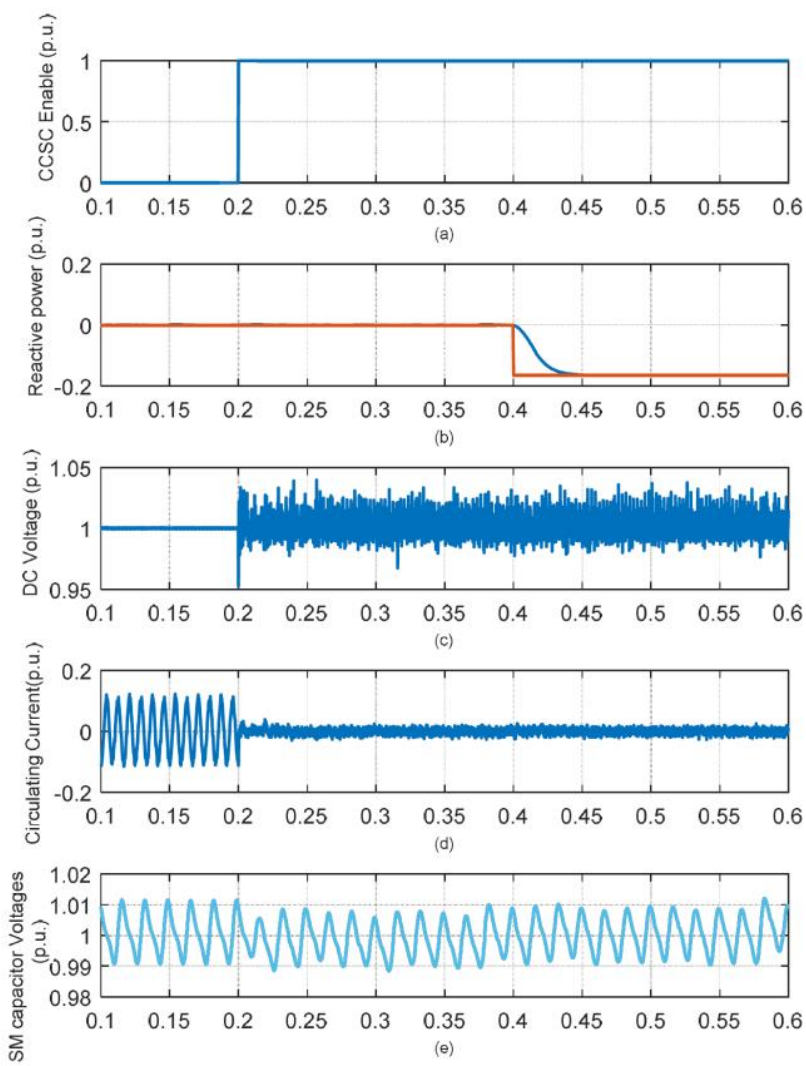

Fig.11. (a) enable signal for CCSC (b) reactive power and reference value (c) DC voltage (d) circulating current (e) SM capacitor voltages in upper arm of phase $A$

\section{Conclusion}

This paper develops the robust Sliding Mode Current Control and Unscented Kalman Filter (UKF) to estimate the capacitor voltages from the measurement of arm 
currents of Modular Multilevel Converter (MMC). UKF use nonlinear unscented transforms in the prediction step in order to preserve the stochastic characteristics of a nonlinear system. In order to design robust control strategy and nonlinear observer, mathematical model of MMC using rotating d-q theory has been used. Digital time-domain simulation studies are carried out in the Matlab/Simulink environment to verify the performance of the overall proposed control system. Simulation results show that the proposed controller is able to track the references of real and reactive power properly and reduce significantly overshoot in the output response.

\section{References}

[1] J. Rodriguez, L. J. Sheng and Z. P. Fang, "Multilevel inverters: a survey of topologies, controls, and applications," Industrial Electronics, IEEE Transactions, vol.49, pp. 724-738, 2002.

[2] M. Hagiwara, K. Nishimura, and H. Akagi, "A MediumVoltage Motor Drive With a Modular Multilevel PWM Inverter," Power Electronics, IEEE Transaction, vol. 25, pp. 1786-1799, 2010.

[3] Yuanze Zhang, Jayashri Ravishankar, John FletcherRui Li, Minxiao Han, "Review of modular multilevel converter based multi-terminal HVDC systems for offshore wind power transmission", Review of modular multilevel converter based multi-terminal HVDC systems for offshore wind power transmission, Vol.61, pp.572-586, 2016.

[4] M. Hagiwara, I. Hasegawa, and H. Akagi, "Start-up and low-speed operation of an electric motor driven by a modular multilevel cascade inverter,'IEEE Trans. Ind. Appl., vol. 49, no. 4, pp. 1556-1565, Jul./Aug. 2013.

[5] A.Hajizadeh, M.A.Golkar, A.Feliachi, "Voltage Control and Active Power Management of Hybrid Fuel-Cell/EnergyStorage Power Conversion System Under Unbalanced Voltage Sag Conditions", IEEE Transactions On Energy Conversion, Vol. 25, No. 4, pp.1195-1208, December 2010.

[6] M. Parker, L. Ran, and S. Finney, "Distributed control of a faulttolerant modular multilevel inverter for direct-drive wind turbine grid interfacing," IEEE Trans. Ind. Electron., vol. 60, no. 2, pp. 509-522, Feb. 2013.

[7] F. Filho, L. Tolbert, Y. Cao, and B. Ozpineci, "Real-time selective harmonic minimization for multilevel inverters connected to solar panels using artificial neural network angle generation," IEEE Trans. Ind. Appl., vol. 47, no. 5, pp. 2117-2124, Jul. 2011.

[8] G. Mondal , R. Critchley , F. Hassan and W. Crookes "Design and simulation of amodular multi-level converter for MVDC application", Proc.2011 IEEE Int. Symp. Ind. Electron. (ISIE), pp.1 -6, 2011.

[9] M. A Parker, L. Ran, and S. J. Finney, "Distributed control of a fault tolerant modular multilevel inverter for directdrive wind turbine grid interfacing," IEEE Trans. Ind. Electron., vol. 60, no. 2, pp. 509-522, Feb. 2013.

[10] S. Sarkka, "On unscented Kalman filtering for state estimation of continuous-time nonlinear systems," IEEE Trans. Autom. Control, vol. 52, no. 9, pp. 1631-1641, Sep. 2007.

[11] A. Jafari Koshkouei, A. S. I. Zinober, "Sliding Mode Control of Discrete-Time Systems", Journal of Dynamic Systems, Measurement, and Control, Vol. 122, pp.793-802, 2000.

[12] T. Orowska-Kowalska, M. Kaminski and K. Szabat, "Implementation of a sliding-mode controller with an integral function and fuzzy gain value for the electrical drive with an elastic joint", IEEE Trans. Ind. Electron., vol. 57, no. 4, pp.1309-1317.2010. 